Supporting Information 


\title{
Multi-scale Photonic Crystal Enhanced Core-Shell Plasmonic Nanomaterial for Rapid Vapor-Phase Detection of Explosives
}

\author{
Kenneth J. Squire ${ }^{1}$, Kundan Sivashanmugan ${ }^{1}$, Boxin Zhang ${ }^{1}$, Erwen $\mathrm{Li}^{1}$, Joseph Kraai ${ }^{2}$, Gregory Rorrer ${ }^{2}$, and Alan \\ X. Wang ${ }^{1^{*}}$ \\ ${ }^{1}$ School of Electrical Engineering and Computer Science, Oregon State University, 1148 Kelley Engineering Center, Corvallis, OR, 97331, USA \\ ${ }^{2}$ School of Chemical, Biological \& Environmental Engineering, Oregon State University, 116 Johnson Hall, Corvallis, OR, 97331, USA \\ *Corresponding author: wang@oregonstate.edu
}

\section{S1 Materials}

All chemicals were used as received. Gold(III) chloride hydrate, (3-Aminopropyl)triethoxysilane (APTES), sodium silicate solution, pyrene and 2,4-dinitrotoluene (DNT) were all purchased from Sigma Aldrich. Polydimethylsiloxane (PDMS) was purchased from Electron Microscopy Sciences. Nitrogen gas was supplied by Airgas. Sodium hydroxide and sodium citrate dihydrate were purchased from Macron and pure ethanol $(\mathrm{EtOH})$ was purchased from our university chemical store. Water used in experiments was deionized and purified with Millipore Synergy UV unit to a resistivity of $\sim 18.2 \mathrm{M} \Omega \mathrm{cm}$.

\section{S2 Frustule Population of Glass Coverslip}

The Pinnularia sp. diatom species were cultured according to the previously described method with minor alterations[1, 2]. Diatoms were obtained from UTEX Culture Collection of Algae (UTEX \#B679) and cultivated in $100 \mathrm{~mL}$ of Harrison's Artificial Seaswater Medium with Guillard's f/2 nutrient enrichment[3]. The cultures were left incubating at $22^{\circ} \mathrm{C}$ and $100 \mu \mathrm{E} / \mathrm{m}^{2} \bullet$ sec for a photocycle of 14 hours light and 10 hours dark and subcultured every 21 days with a 10:1 dilution in fresh medium. When harvested, cell densities were $\sim 10^{6}$ cells $/ \mathrm{mL}$ as measured using a Beckman Coulter Z2 Particle Counter with $6 \mu \mathrm{m}$ threshold and $100 \mu \mathrm{L}$ of cell suspension being diluted in $10 \mathrm{~mL}$ diluent (10 g/L sterile-filtered $\mathrm{NaCl}, 171$ $\mathrm{mM})$.

The method of isolation was described in previous work with minor adaptations[1, 2]. The diatoms were grown for three weeks and allowed to settle to the bottom before being decanted and centrifuged for $20 \mathrm{~min}$ at $2500 \mathrm{~g}$. $40 \mathrm{~mL}$ of nanopore water was used to wash the packed cells three times to remove salts from the culture medium, after which the cells were re-suspended in $1 \mathrm{~mL}$ of $8.1 \mathrm{M} \mathrm{HCl}$ and left gently mixing. Again, the diatoms settled and supernatant was removed. Oxidation was performed by adding $5 \mathrm{~mL}$ of concentrated diatoms to $32 \mathrm{~mL}$ of $0.76 \mathrm{HCl}$ in $28 \%(\mathrm{v} / \mathrm{v}) \mathrm{H}_{2} \mathrm{O}_{2}$. This was mixed then left to sit for 72 hours with daily mixing. This oxidation process was performed twice followed by supernatant removal and diatom resuspension in $40 \mathrm{~mL} \mathrm{H}_{2} \mathrm{O}$. The diatoms settled, and the supernatant was removed. This aqueous washing was repeated twice more before being suspended in anhydrous methanol. A similar washing was performed with methanol a total of three times and the isolated diatom frustules were stored in methanol at room temperature.

To populate a coverslip with diatom frustules, it was first sonicated in $1 \%(\mathrm{v} / \mathrm{v})$ solution of Liquinox detergent for 60 minutes at $40^{\circ} \mathrm{C}$. It was rinsed with Nanopure $\mathrm{H}_{2} \mathrm{O}$ and $100 \%$ ethanol. The coverslip was dried with nitrogen gas then put in a UV-Ozone Cleaner for 30 minutes at $90^{\circ} \mathrm{C}$. The coverslips were placed in a petri dish set upon a hot plate at $32^{\circ} \mathrm{C}$. $100 \mu \mathrm{L}$ of $0.24 \mathrm{mg} / \mathrm{mL}$ frustule suspension in ethanol was dispersed onto the surface and allowed to evaporate. The frustule-populated coverslips were placed in 
the UV-Ozone Cleaner again for 24 hours at $90^{\circ} \mathrm{C}$ to oxidize any remaining organic contaminants and fix the diatoms to the substrate. Substrates were stored for future use.

\section{S3 Multi-Scale SERS Substrate Assembly}

The frustule populated coverslip was diced into $~ 5 \times 5 \mathrm{~mm}$ pieces. $1 \mu \mathrm{L}$ of $\mathrm{Au} @ \mathrm{SiO}_{2}$ nanoparticle (NP) solution was dropcast onto the substrate and dried at $60^{\circ}$. This is represented in Fig. 2 in the main text and was repeated for a total of four drops.

\section{S4 Characterization Equipment}

The following equipment was used in the characterization of our hybrid SERS substrate. A Thermo Fisher Scientific Nanodrop 2000C Spectrophotometer was used for adsorption measurements of the colloidal coreshell NPs and the Sorvall Legend XI Centrifuge was used to concentrate the NPs. FEI Quanta 600F SEM and Titan 80-200 TEM were used for scanning and transmission electron microscopy respectively. Energydispersive X-ray spectroscopy (EDS) was also performed on the Titan 80-200.

\section{S5 Raman Measurements}

Raman measurements were obtained using a B\&W Tek i-Raman Plus 785 nm portable Raman spectrometer with an $85 \mu \mathrm{m}$ beam diameter. For pyrene characterization measurements, 20 second integration time with $100 \mathrm{~mW}$ power was used. DNT application measurements were obtained using 60 second integration with $50 \mathrm{~mW}$ power.

\section{S6 Nanoparticle Characterization}

A representative SEM image of core-shell NPs is shown in Fig. S1a. The NP size distribution was obtained using ImageJ on 174 particles and the histogram is shown in Fig. S1b. Absorption measurements were taken and shown in Fig. S1c below. Strong absorption was measured, indicating the plasmonic capability of the nanoparticles.
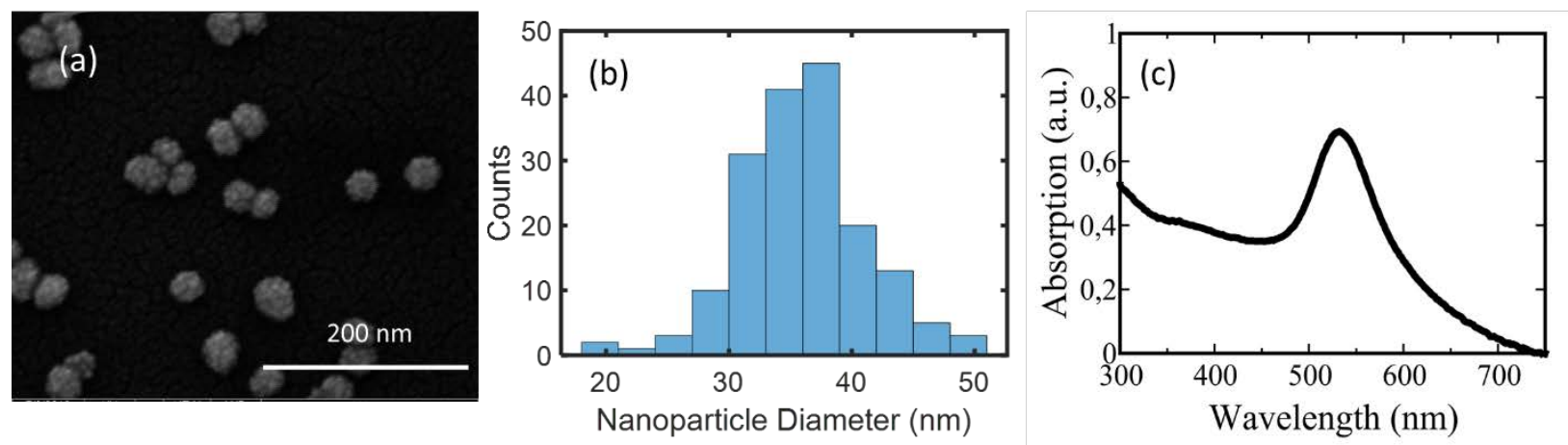

Fig. S1: Representative SEM image of core-shell NPs (a). Plots showing NP size distribution (b) and the absorption spectrum of core-shell NPs (c).

\section{S7 Au@SiO 2 EDS Characterization}



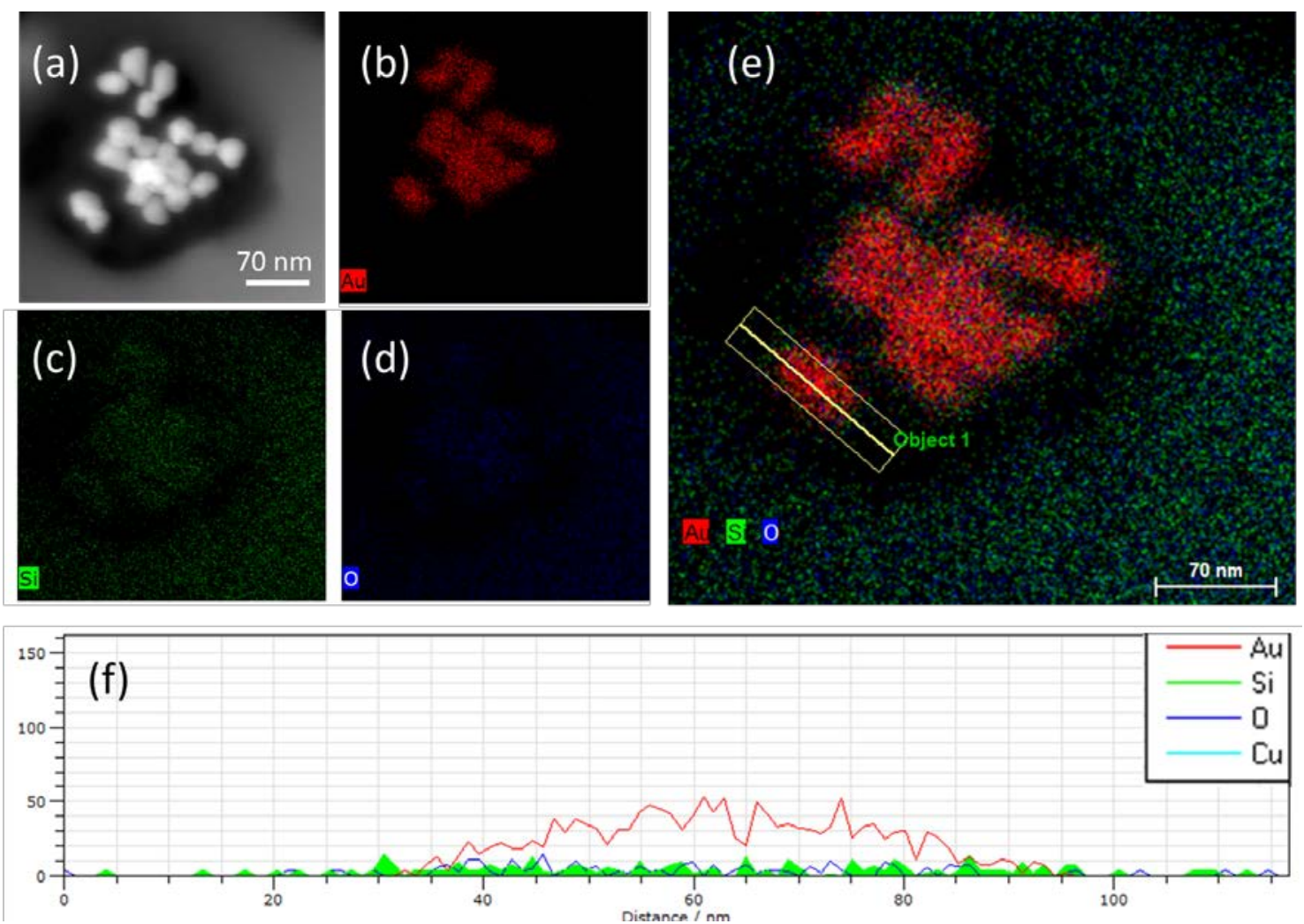

Fig. S2: TEM image of $\mathrm{Au@SiO}{ }_{2} \mathrm{NPs}$ in frustule pore is shown (a). EDS was performed and the components are split into Au (b), Si (c) and O (d) as well as all three combined (e). A cross-sectional line map was created, and the composition is shown in (f) clearly showing the spatial separation of the Au core and the Si shell.

\section{S8 SERS Capabilities of Au@SiO 2 NPs}

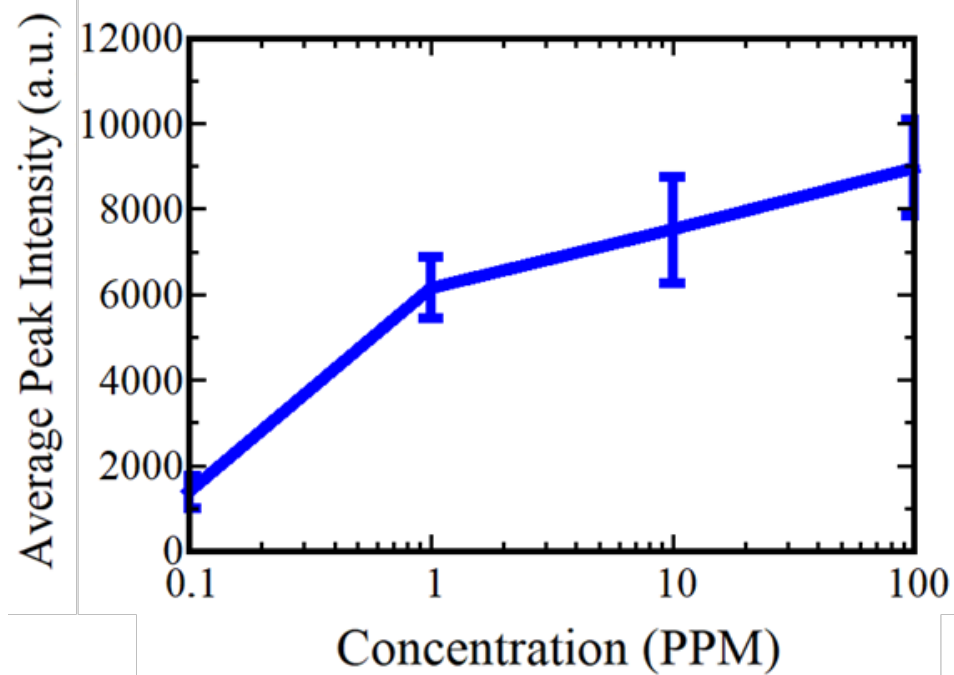

Fig. S3: SERS of pyrene dropcast on frustule-Au@SiO 2 substrates to show potential of core-shell NPs for SERS measurement. 


\section{S9 Nanoparticle Distribution on Diatom and Glass Substrates}
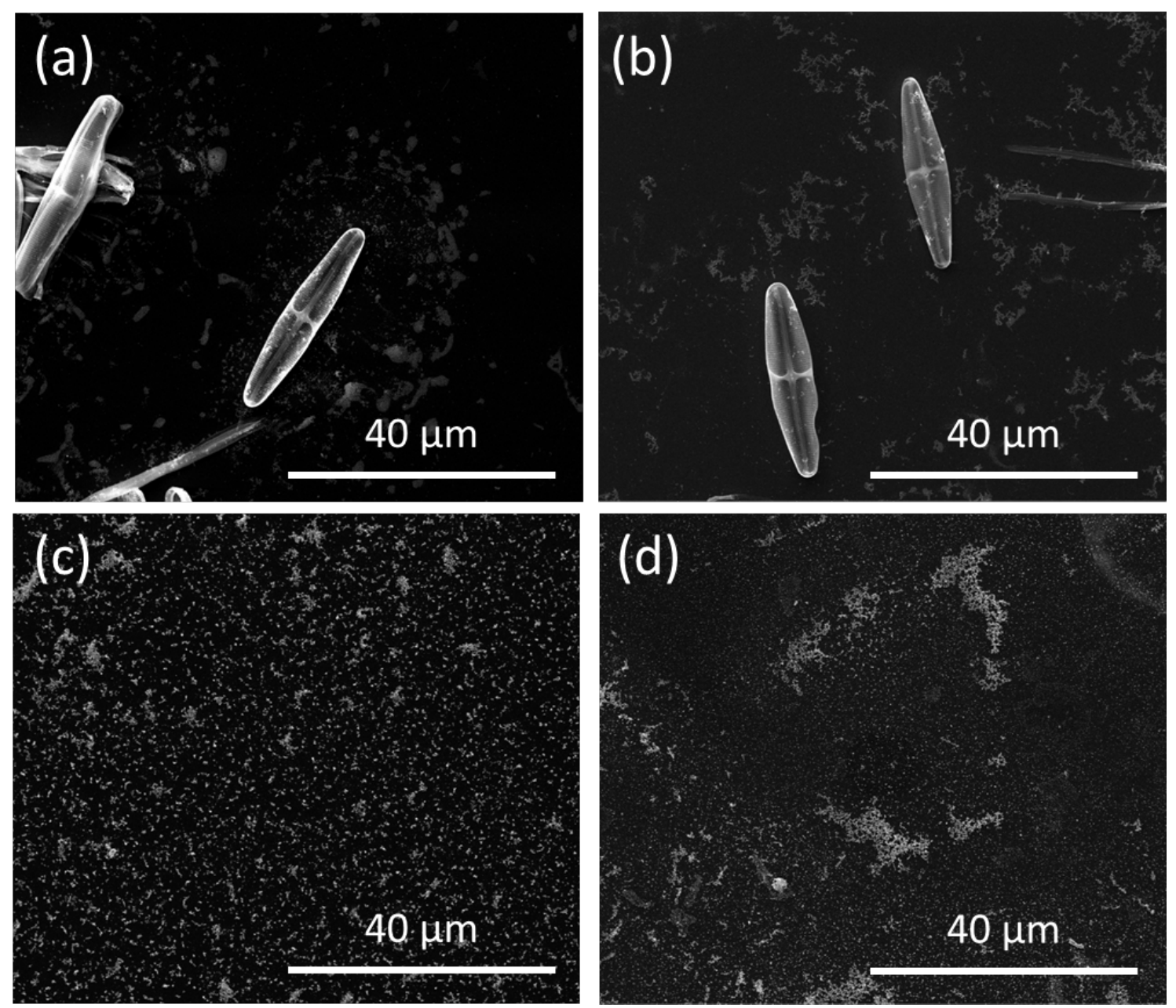

Fig. S4: SEM images of diatom frustule (a,b) and glass (c,d) substrates with core-shell NPs (a,c) and Au NPs (b,d) with a horizontal field width of $85 \mu \mathrm{m}$ corresponding to the size of the laser spot. This clearly shows the higher density of NPs on glass despite which, the SERS intensity on diatom is superior.

\section{S10 Reproducibility}

To verify the reproducibility of the setup and sensors, three diatom-populated substrates with three drops of $\mathrm{Au} @ \mathrm{SiO}_{2} \mathrm{NPs}$ were placed in vapor chambers with $20 \mu \mathrm{L}$ of 1000 ppm pyrene in EtOH. Each sample was left heating at $70^{\circ}$ for 45 minutes and then immediately removed and measured. Similar to the other measurements, 20 spectra were measured, the characteristic peak intensity was calculated and the 10 spectra with intensities closest to the mean were used. The average characteristic peak intensity was then calculated, as well as the standard deviation. The three normalized peak intensities for the samples were plotted below in Fig. S5 with the standard deviation. From this plot, it is easy to see the reproducibility of these SERS substrates. 


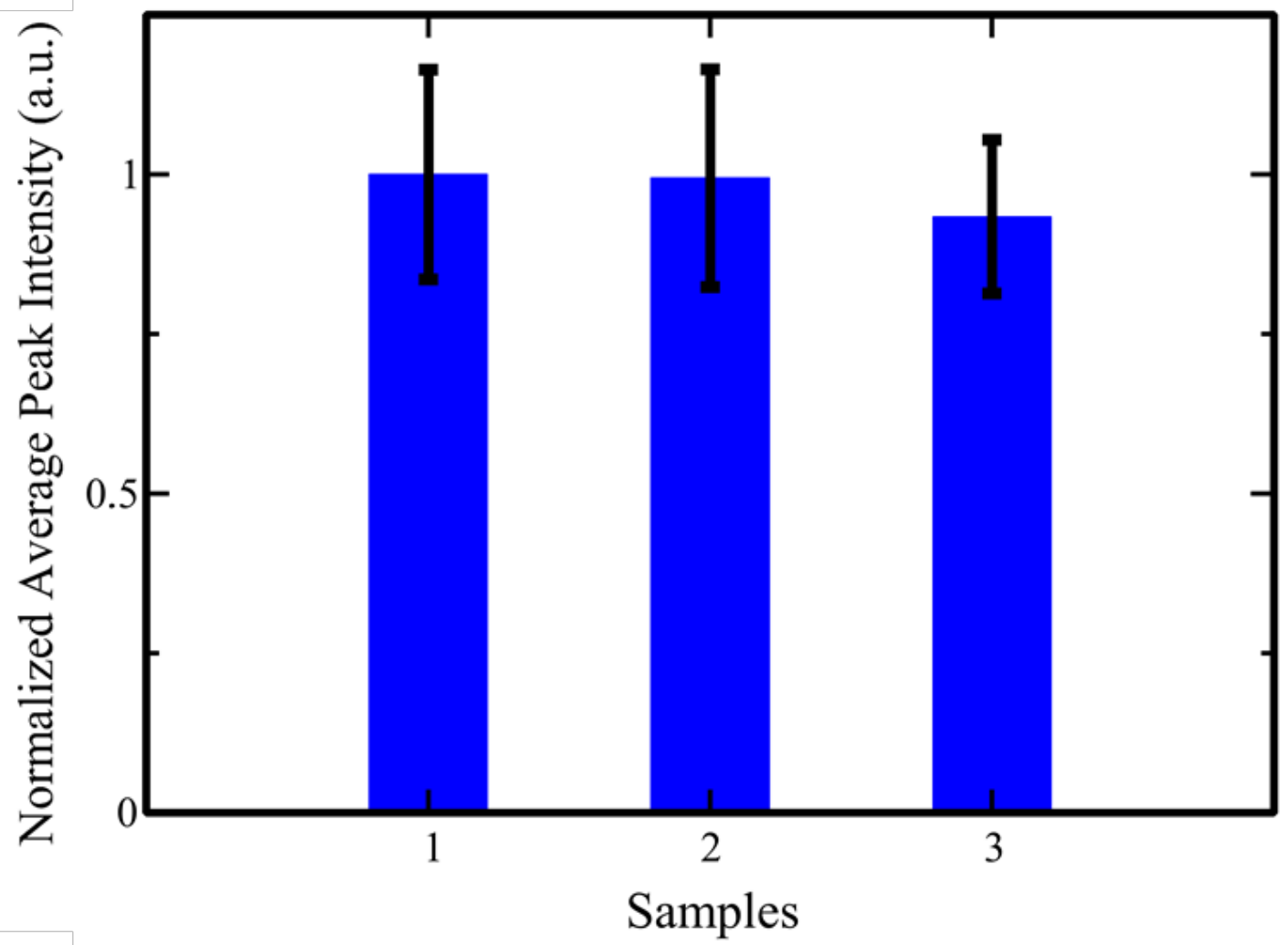

Fig. S5: Normalized average peak intensity of three samples performed under the same circumstances to demonstrate the reproducibility of the substrates. 


\section{References:}

[1] C. Jeffryes, T. Gutu, J. Jiao, G.L. Rorrer, Two-stage photobioreactor process for the metabolic insertion of nanostructured germanium into the silica microstructure of the diatom Pinnularia sp, Materials Science and Engineering: C, 28(2008) 107-18.

[2] J.A. Kraai, G.L. Rorrer, A.X. Wang, Highly-porous diatom biosilica stationary phase for thin-layer chromatography, Journal of Chromatography A, 1591(2019) 162-70.

[3] J.A. Berges, D.J. Franklin, P.J. Harrison, Evolution of an artificial seawater medium: improvements in enriched seawater, artificial water over the last two decades, Journal of Phycology, 37(2001) 1138-45. 